\title{
Complete Pharyngeal Clearance Following Swallowing
}

National Cancer Institute

\section{Source}

National Cancer Institute. Complete Pharyngeal Clearance Following Swallowing. NCI

Thesaurus. Code C127331.

A finding of complete pharyngeal clearance following swallowing. 